\title{
Alkaline phosphatase downregulation promotes lung adenocarcinoma metastasis via the c-Myc/RhoA axis
}

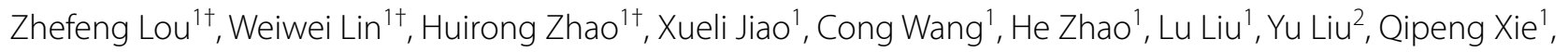
Xing Huang ${ }^{3^{*}}$, Haishan Huang ${ }^{1 *}$ and Lingling Zhao ${ }^{*^{*}}$ (D)

\begin{abstract}
Background: Lung adenocarcinoma (LUAD) metastasis significantly reduces patient survival; hence inhibiting the metastatic ability of lung cancer cells will greatly prolong patient survival. Alkaline phosphatase (ALPL), a homodimeric cell surface phosphohydrolase, is reported to play a controversial role in prostate cancer and ovarian cancer cell migration; however, the function of ALPL in LUAD and the related mechanisms remain unclear.

Methods: TCGA database was used to analysis the expression of ALPL, and further verification was performed in a cohort of 36 LUAD samples by qPCR and western blot. Soft-agar assay, transwell assay and lung metastasis assay were employed to detect the function of ALPL in LUAD progression. The $\mathrm{gPCR}$, luciferase promoter reporter assay and western blot were used to clarify the molecular mechanisms of ALPL in promoting metastasis in LUAD.

Results: ALPL was downregulated in LUAD, and the disease-free survival rate of patients with low ALPL was significantly reduced. Further studies showed that overexpression of ALPL in LUAD cell lines did not significantly affect cell proliferation, but it did significantly attenuate lung metastasis in a mouse model. ALPL downregulation in LUAD led to a decrease in the amount of phosphorylated (p)-ERK. Because p-ERK promotes the classical c-Myc degradation pathway, the decrease in p-ERK led to the accumulation of c-Myc and therefore to an increase in RhoA transcription, which enhanced LUAD cell metastasis.
\end{abstract}

Conclusion: ALPL specially inhibits the metastasis of LUAD cells by affecting the p-ERK/C-Myc/RhoA axis, providing a theoretical basis for the targeted therapy of clinical LUAD.

Keywords: ALPL, Lung adenocarcinoma (LUAD), C-Myc degradation, RhoA, Metastasis

*Correspondence: huangxing66@zju.edu.cn; haishan_333@163.com; zhaolingling@wmu.edu.cn

'Zhefeng Lou, Weiwei Lin and Huirong Zhao contributed equally to this work

${ }^{1}$ Zhejiang Provincial Key Laboratory of Medical Genetics, Key Laboratory of Laboratory Medicine, Ministry of Education, School of Laboratory Medicine and Life Sciences, Wenzhou Medical University, Wenzhou 325035, Zhejiang, China

${ }^{3}$ Zhejiang Provincial Key Laboratory of Pancreatic Disease, School of Medicine, the First Affiliated Hospital, Zhejiang University, Hangzhou 310003, Zhejiang, China

Full list of author information is available at the end of the article

\section{Background}

According to the Global Cancer Analysis Data for 2020, lung cancer accounts for $11.4 \%$ of all cancers and has an overall mortality rate of $18.0 \%$. Its morbidity and mortality rates are the highest of the major cancers [1]. Lung cancer is classified as small cell lung cancer (SCLC) or non-small cell lung cancer (NSCLC) according to the tissue type [2]. NSCLC comprises three main subtypes, lung adenocarcinoma (LUAD), lung squamous cell carcinoma (LUSC), and large cell carcinoma. LUAD accounts for approximately $40-50 \%$ of NSCLC cases and is thus

(c) The Author(s) 2021. This article is licensed under a Creative Commons Attribution 4.0 International License, which permits use, sharing, adaptation, distribution and reproduction in any medium or format, as long as you give appropriate credit to the original author(s) and the source, provide a link to the Creative Commons licence, and indicate if changes were made. The images or other third party material in this article are included in the article's Creative Commons licence, unless indicated otherwise in a credit line to the material. If material is not included in the article's Creative Commons licence and your intended use is not permitted by statutory regulation or exceeds the permitted use, you will need to obtain permission directly from the copyright holder. To view a copy of this licence, visit http://creativeco mmons.org/licenses/by/4.0/. The Creative Commons Public Domain Dedication waiver (http://creativecommons.org/publicdomain/ zero/1.0/) applies to the data made available in this article, unless otherwise stated in a credit line to the data. 
the main pathological type of lung cancer [3]. Clinically, more than $60 \%$ of patients present with later-stage (stage III or IV) disease, which contributes to the poor prognosis of LUAD [3]. Furthermore, the data for 2007-2013 in the USA showed that the 5 year survival rate for lung cancer decreases from 56 to $29 \%$ to $5 \%$ as the disease progresses from localized to regional and then to distant, respectively [4], indicating that lung cancer metastasis significantly reduces patient survival. Therefore, inhibiting the metastatic ability of lung cancer cells will greatly prolong patient survival.

Studies of the gene encoding alkaline phosphatase (ALPL) are focused mainly on hypophosphatemia. Various mutations in this gene have been shown to affect its activity and cause a decrease in serum ALPL levels, resulting in hypercalcemia and hypokalemia [5]. ALPL plays a crucial role in intracellular signaling and regulation of protein activity [6-8]. Dysfunction of this enzyme is related to the development of multiple diseases, including diabetes mellitus, liver disease, stork and Alzheimer's disease [9-12]. According to recent reports, ALPL also plays a role in cancer. Rao et al. found that knockdown of ALPL can reduce prostate cancer cell migration [10]. Another study suggested that overexpression of ALPL can inhibit the migration and invasion of HGSOC ovarian cancer cells [13]. Therefore, the function of ALPL in cancer and the related mechanisms remain controversial.

c-Myc is one of the most well-characterized transcription factors in tumors. Analysis of a hybrid of rodent and human Burkitt lymphoma cells revealed the presence of a reciprocal translocation between chromosomes 8 and 14 that disrupted the $M Y C$ gene [14], which confirmed that $M Y C$ functions as an oncogene in humans [15]. Translocations in $M Y C$ occur often in hematopoietic cancers, and it was reported to be the third most frequently amplified gene in human cancers in a whole-genome copy number analysis $[16,17]$. Its downstream effector, RhoA, is a member of the Rho family of small GTPases and is a key regulator of actin polymerization and cell migration $[18,19]$. It acts as a molecular switch in cellular processes such as cell morphogenesis, adhesion, and migration $[20,21]$. The primary function of RhoA is to remodel the actin cytoskeleton during cellular processes such as migration and endocytosis, which also affects the local dynamics of microtubules (MTs) [22]. Thus, RhoA clearly plays an important role in cell migration and invasion $[23,24]$.

In this study, we revealed a molecular mechanism through which ALPL regulates LUAD invasion and metastasis by affecting the c-Myc/RhoA cascade. These findings provide a theoretical basis for the targeted therapy of clinical LUAD.

\section{Methods}

\section{Plasmids and antibodies}

The Plvx-GFP plasmid was purchased from Sunny Biotechnology (Shanghai, China). The Plvx-GFP-ALPL expression plasmid was constructed using the following primers: forward: 5'-CCG CTC GAG GCC ACC ATG ATT TCA CCA TTC TTA GTA CTG GCC - $3^{\prime}$, reverse: $5^{\prime}$-CCG GAA TTC GGT GAA CAG GAC GCT CAG GGG-3'. The Plvx-GFP-RhoA expression plasmid was constructed using the following primers: forward: 5'-CCG CTC GAG GCC ACC ATG GCT GCC ATC CGG AAG A-3', reverse: $5^{\prime}$-CCG GAA TTC ACC CAA GAC AAG GCA CCC AGA T-3'. The RhoA promoter-driven luciferase reporter was constructed into PGL3 Basic vector using primers, forward: 5'-CGG GGT ACC ACT TCC TGT ATC CTG TTG TTT GTG T $-3^{\prime}$, reverse: $5^{\prime}$-CCC AAG CTT CAA ATG ACA ATG ACA CAG GAC ATA C - $3^{\prime}$. The c-Myc plasmid was from our research group [25]. The antibody against ALPL (GTTX100817) was purchased from Genetex (Irvine, CA, USA). The antibodies against RhoA (2117S), ERK1/2 (4695S), and p-ERK1/2 (4370S) were purchased from Cell Signaling Technology (Beverly, MA, USA). The antibodies against c-Myc (sc-764), RhoA (sc-418) and p-c-Myc (sc-377552) were purchased from Santa Cruz Biotechnology (Santa Cruz, CA, USA). The antibody against GAPDH (GTX100118) was purchased from Genetex. 10X Cell Lysis Buffer (9803) was purchased from Cell Signaling Technology.

\section{Cell culture and transfection}

Normal bronchus epithelial cell line Beas-2B and lung cancer cell lines A549, H1975, HCC827, H1299, and SKMES-1 were purchased from the American Type Culture Collection (ATCC; Manassas, VA, USA). All cells were subjected to DNA analysis and authenticated before use in these studies [26]. A549 and SK-MES-1 cells were cultured in F12K (21127-022; Gibco/ThermoFisher Scientific, Waltham, MA, USA) supplemented with $10 \%$ fetal bovine serum (FBS) (10437-028; Gibco). H1975 cells were cultured in a 1:1 mixture of DMEM/Ham's F12 medium (10565-018; Gibco) supplemented with 5\% FBS. HCC827 and H1299 cells were cultured in RPMI 1640 (11875-093; Gibco) supplemented with 10\% FBS, and Beas-2B cells were cultured in DMEM (11995-065; Gibco) supplemented with $10 \%$ FBS. Cell transfections were performed using PolyJet DNA In Vitro Transfection Reagent (SignaGen Laboratories, Rockville, MD, USA) according to the manufacturer's instructions. For stable cell line selection, cells were treated with G418 $(500-1000 \mu \mathrm{g} / \mathrm{mL})$ or puromycin $(0.2-0.3 \mu \mathrm{g} / \mathrm{mL})$ depending on the antibiotic 
resistance plasmid used. Cells surviving the antibiotic selection were pooled as stable mass transfectants [27].

\section{Reverse transcription-PCR}

Total RNA was extracted using TRIzol reagent (Invitrogen/ThermoFisher Scientific) according to the manufacturer's instructions. Next, $5 \mu \mathrm{g}$ total RNA was used for first-strand cDNA synthesis with the SuperScript First-Strand Synthesis system and oligo (dT) primers (Invitrogen), as described previously [28].

\section{Quantitative RT-PCR}

Fast SYBR Green Master Mix (4385614; Applied Biosystems/ThermoFisher Scientific) was used for realtime PCR with the 7900HT Fast Real-Time PCR system (Applied Biosystems) [29]. The primers used in this study were as follows: human ALPL (forward: $5^{\prime}-\mathrm{AC}$ CGA GAT ACA AGC ACT CCC ACT-3', reverse: $5^{\prime}$ CC GTC ACG TTG TTC CTG TTC AG $3^{\prime}$ ), human RhoA (forward: $5^{\prime}$-ACT ATG TGG CAG ATA TCG AGG TGG A $3^{\prime}$, reverse: $5^{\prime}$ - TA TCA GGG CTG TCG ATG GAA AAA C-3'), human c-Myc (forward: 5'-GGA GGA ACA AGA AGA TGA GGA AGA AA - $3^{\prime}$, reverse: $5^{\prime}$-TGA GGA CCA GTG GGC TGT GAG G-3'), and human GAPDH (forward: 5'-GAC TCA TGA CCA CAG TCC ATG C-3', reverse: $5^{\prime}$-CAG GTC AGG TCC ACC ACT GA-3').

\section{Western blot analysis}

Whole cell extracts were prepared in cell lysis buffer $[10 \mathrm{mM}$ Tris- $\mathrm{HCl}$ (pH 7.4), $1 \%$ SDS, and $1 \mathrm{mM}$ $\mathrm{Na}_{3} \mathrm{VO}_{4}$ ]. Western blotting was performed as described previously $[27,30]$. Briefly, blots were incubated with primary antibodies diluted 1:500-1:1000 in 5\% BSA for $12-16 \mathrm{~h}$ at $4{ }^{\circ} \mathrm{C}$, and then incubated with secondary antibody diluted 1:1000-1:2000 in 5\% skim milk for $2-3 \mathrm{~h}$ at $4{ }^{\circ} \mathrm{C}$. The images were acquired by scanning with the Phosphoimager Typhoon FLA 7000 (GE, Pittsburgh, PA, USA) [27].

\section{Soft-agar assay}

The anchorage-independent growth ability of the cells was evaluated in soft agar, as described [30]. Briefly, $3 \mathrm{~mL}$ of $0.5 \%$ agar (214010; Becton Dickinson) in basal modified Eagle's medium supplemented with $10 \%$ FBS was layered onto each well of 6-well tissue culture plates. Cells $\left(3 \times 10^{4}\right)$ suspended in $1 \mathrm{~mL}$ normal medium (B9638; Sigma-Aldrich) were mixed with $2 \mathrm{~mL}$ of $0.5 \%$ agar in basal modified Eagle's medium supplemented with $10 \% \mathrm{FBS}$, and $1 \mathrm{~mL}$ of the mixture was added into each well on top of the $0.5 \%$ agar layer. Plates were incubated at $37{ }^{\circ} \mathrm{C}$ in $5 \% \mathrm{CO}_{2}$ for 3 weeks, and the colonies were counted. The data is presented as the number of colonies $/ 10^{4}$ cells [30].

\section{Cell migration and invasion assay}

Cell migration and invasion were evaluated using kits from BD Falcon. The assay was performed according to the manufacturer's instructions, as described previously $[27,31]$. Briefly, cells were seeded in the inserts in $400 \mu \mathrm{L}$ serum-free medium. The inserts were placed in wells containing $700 \mu \mathrm{L}$ medium with $10 \%$ FBS and incubated for $24 \mathrm{~h}$. Then, cells on the upper surface of the filters were removed with a cotton swab. The inserts were fixed in methanol and stained with Giemsa, imaged with a microscope, and then the cells were counted. The data shown are representative of three independent experiments [32].

\section{Tube formation assay}

Tube formation assay was conducted on HUVECs under the treatment of different LUAD cancer cell culture medium. HUVECs $\left(2.0 \times 10^{4}\right.$ cells $)$ were cultured in the presence of the conditioned medium in 96-well plates coated with Matrigel $(50 \mu \mathrm{L} /$ well, BD Biosciences). After $4 \mathrm{~h}$ incubation at $37^{\circ} \mathrm{C}$, tube formation was visualized under light microscope. Capillary length was calculated with Image J software. This assay was carried out in triplicate.

\section{Lung metastasis assay}

Female euthymic $(\mathrm{nu}+/ \mathrm{nu}+)$ mice were purchased from Shanghai Silaike Experimental Animal Company (license no. SCXK, Shanghai 2010 0002; Shanghai, China). At age 4-5 weeks, the mice were randomized and injected with cells [A549 (vector), A549 (ALPL), HCC827 (vector), and HCC827 (ALPL)] via the lateral tail vein $\left(3 \times 10^{6}\right.$ cells in $100 \mu \mathrm{L}$ PBS/mouse). Detailed experimental methods have been described previously [27].

\section{Luciferase promoter reporter assay}

The RhoA luciferase reporter and pRL-TK were each transiently co-transfected into A549 and HCC827 cells. Luciferase activity was determined $24 \mathrm{~h}$ after transfection using the Luciferase Assay System (Promega, Madison, WI, USA), as described [27, 33, 34]. The values were normalized to the internal thymidine kinase (TK) signal. All experiments were conducted in triplicate, and the results were expressed as the mean \pm standard error [27]. 


\section{H\&E staining}

Fixed lung tissues were dehydrated using an alcohol gradient, paraffin-embedded, and then stained with $H \& E$, as described in detail previously [35]. Images were captured using the Nikon Eclipse Ni microsystem (Nikon DS-Ri2; Tokyo, Japan).

\section{Immunohistochemistry (IHC)}

IHC assays were performed to detect the expression of ALPL, p-ERK, c-Myc and RhoA in formalin-fixed paraffin-embedded metastatic lung tumor tissues of mice injected with LUAD cells. Specific antibodies against ALPL (GTTX100817, Genetex), p-ERK1/2 (4370S, CST), c-Myc (sc-764, Santa Cruz) and RhoA (sc-418, Santa Cruz) were used for IHC staining, which were performed using a kit from Boster Bio-Engineering Company (SA1022; Wuhan, China). Immunostained images were captured with the Nikon Eclipse Ni microsystem (DS-Ri2) and analyzed with Image-Pro Plus version 6.0 (Media Cybernetics, Rockville, MD, USA) by calculating the integrated optical density (IOD) of each stained area (IOD/area). At least five images per specimen were counted.

\section{Clinical specimens}

In total, 36 pairs of human LUAD tissues and corresponding adjacent normal tissues were obtained. The authorized case information is shown in Additional file 1: Table S1. Preoperative biopsy and postoperative pathological examination of the above patients confirmed LUAD. The tissue samples were snap-frozen in liquid nitrogen at the time of surgery, RNA was extracted, and cDNA was synthesized and stored at $-80^{\circ} \mathrm{C}$.

\section{Statistical analysis}

All experimental data are expressed as the mean \pm standard deviation (mean $\pm \mathrm{SD}$ ). Graphs and statistical analyses were performed using GraphPad Prism 6 (GraphPad Software, San Diego, CA, USA). The Kaplan-Meier method was used to draw the survival curve, and the logrank test was used to compare the differences between groups. Comparisons between the control group and the experimental group were performed using the Student's t-test. Correlation between two independent groups were performed using the Pearson's Chi-square test. $P<0.05$ was considered statistically significant.
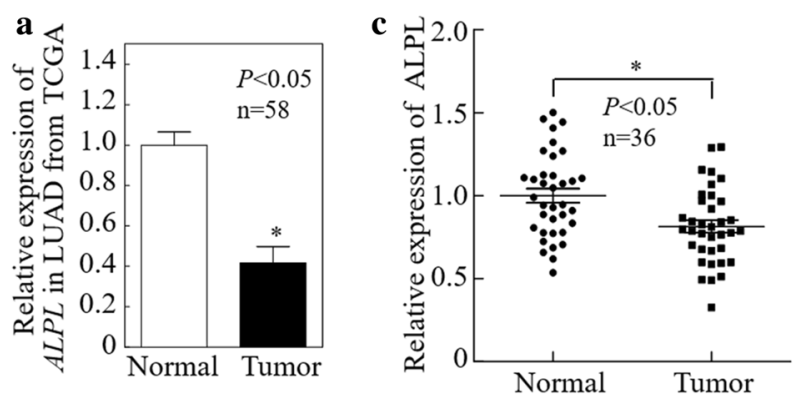

e
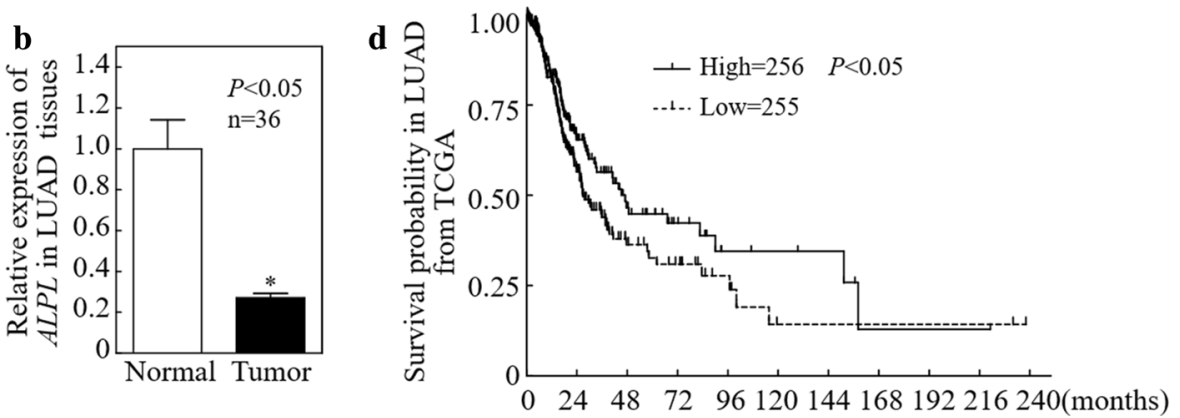

Fig. 1 Alkaline phosphatase (ALPL) is downregulated in LUAD. a ALPL mRNA expression was analyzed in the 58 paired LUAD cases in the Cancer Genome Atlas (TCGA). b ALPL mRNA expression was analyzed in 36 paired fresh clinical LUAD tissues. c Western blot analysis of total protein lysates prepared from 36 paired fresh clinical LUAD tissues. Then perform grayscale analysis and statistics. $\mathbf{d}$ The relationship between ALPL expression in LUAD patients in the TCGA database and the disease-free survival rate. e Western blot analysis of ALPL protein levels in LUAD cell lines. GAPDH was used as an internal control. All results were presented as the mean \pm SD and analyzed by Student's $t$-test. The survival curve was analyzed by log-rank test. Asterisks $\left(^{*}\right)$ represent statistical significance $(P<0.05)$ 


\section{Results}

\section{ALPL is downregulated in LUAD and related to patient} prognosis

Analysis of the 58 paired LUAD cases in the Cancer Genome Atlas (TCGA) database revealed significantly lower expression of ALPL mRNA in LAUD tumors compared with matched healthy lung tissues (Fig. 1a). A separate qPCR analysis of 36 fresh LUAD samples and matched healthy tissues revealed significantly lower ALPL expression in tumor tissues, consistent with the results obtained using the TCGA database (Fig. 1b). Furthermore, western blot experiments were performed on 36 paired LUAD samples, and grayscale analysis was used to make statistics. The results showed that compared with matched healthy tissues, the expression of ALPL protein in LUAD tumors was significantly down-regulated (Fig. 1c, Additional file 1: Fig. S1). Most important, a survival analysis of LUAD patients in the TCGA database revealed that patients with low expression of ALPL had a significantly worse prognosis (Fig. 1d). We therefore concluded that ALPL
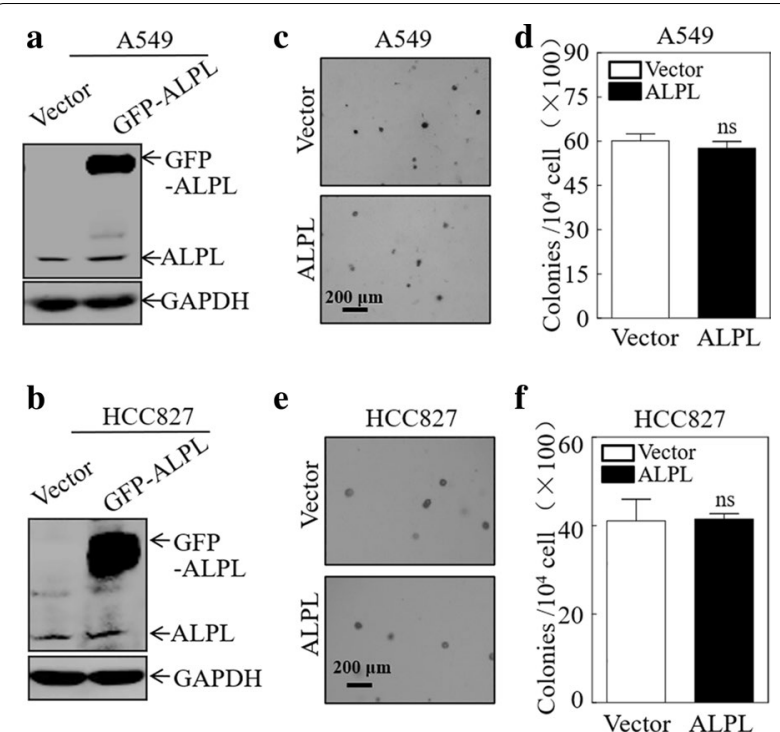

Fig. 2 Alkaline phosphatase (ALPL) has no effect on the proliferation of LUAD cells. Western blot analysis of A549 (a) and HCC827 (b) cells stably transfected with an ALPL overexpression plasmid or a control plasmid. GAPDH was used as an internal control. Soft-agar assay to determine the effect of ALPL overexpression on anchorage-independent growth of A549 (c, d) and HCC827 (e, f) cells. Representative images of colonies were captured by microscopy after 3 weeks of incubation (c, e), Scale bars: $200 \mu$ m. The number of colonies with $>32$ cells were counted in A549 (Vector) and A549 (ALPL) cells (d), and in HCC827 (Vector) and HCC827 (ALPL) cells (f). The results represent the number of colonies per 10,000 cells. Data were presented as the mean \pm SD and analyzed by Student's t-test, $\mathrm{n}=3$. ns, no statistical significance relative to the vector control cells $(P>0.05)$ downregulation in LUAD might be an important molecular event for cancer development or progression. Then we examined the protein expression of ALPL in normal lung cells and LUAD cells, and the results showed that the expression of ALPL in LUAD cells was lower than that in normal lung cells (Fig. 1e).

\section{ALPL has no effect on LUAD cell proliferation}

To investigate the function of ALPL in LUAD cells, we selected two LUAD cell lines, A549 and HCC827, which have relatively low expression of ALPL, and established cell lines stably overexpressing ALPL (Fig. 2a, b). A human lung squamous cell carcinoma (LUSC) cell line (SK-MES-1) stably overexpressing ALPL was also generated (Additional file 1: Fig. S2a). To assess the effects of ALPL on LUAD cell growth, soft-agar experiments were carried out. ALPL overexpression had no effect on the anchorage-independent growth of LUAD (Fig. 2c-f) or LUSC cells (Additional file 1: Fig. S2b, c).

\section{ALPL significantly inhibits the migration and invasion of LUAD cells, and represses LUAD metastasis in a xenograft model}

The prognosis of lung cancer is significantly worse once it has metastasized. Evaluation of the TCGA database revealed higher levels of $A L P L$ in patients with stage I LAUD compared with patients with later-stage disease (II, III, and IV; Additional file 1: Fig. S3). This suggested that ALPL expression may be closely related to LUAD metastasis. To evaluate this, we performed Transwell and wound healing experiments to investigate the effects of ALPL on LUAD cell migration and invasion. ALPL overexpression significantly inhibited the migration and invasion of both A549 and HCC827 cells (Fig. 3a-d, Additional file 1: Fig. S4). By contrast, ALPL overexpression had no effect on the migration or invasion of LUSC cells (SK-MES-1; Additional file 1: Fig. S2d, e). This result may explain the observation that the survival of LUSC patients in the TCGA database was not related to ALPL expression (Additional file 1: Fig. S5). The effect of ALPL on the metastatic capacity of LUAD cells was verified in vivo in a nude mouse xenograft model of lung cancer metastasis. ALPL overexpression significantly reduced the number and size of A549 lung cancer metastases (Fig. 3e-g, Additional file 1: Fig. S6). ALPL overexpression had the same effect in HCC827 cells (Additional file 1: Fig. S7a-c). Moreover, the results of tube formation assay showed that ALPL overexpression also inhibited angiogenesis (Fig. 3h, i). Therefore, in both in vivo and in vitro experiments, ALPL overexpression specially inhibited the migration of LUAD cells. 

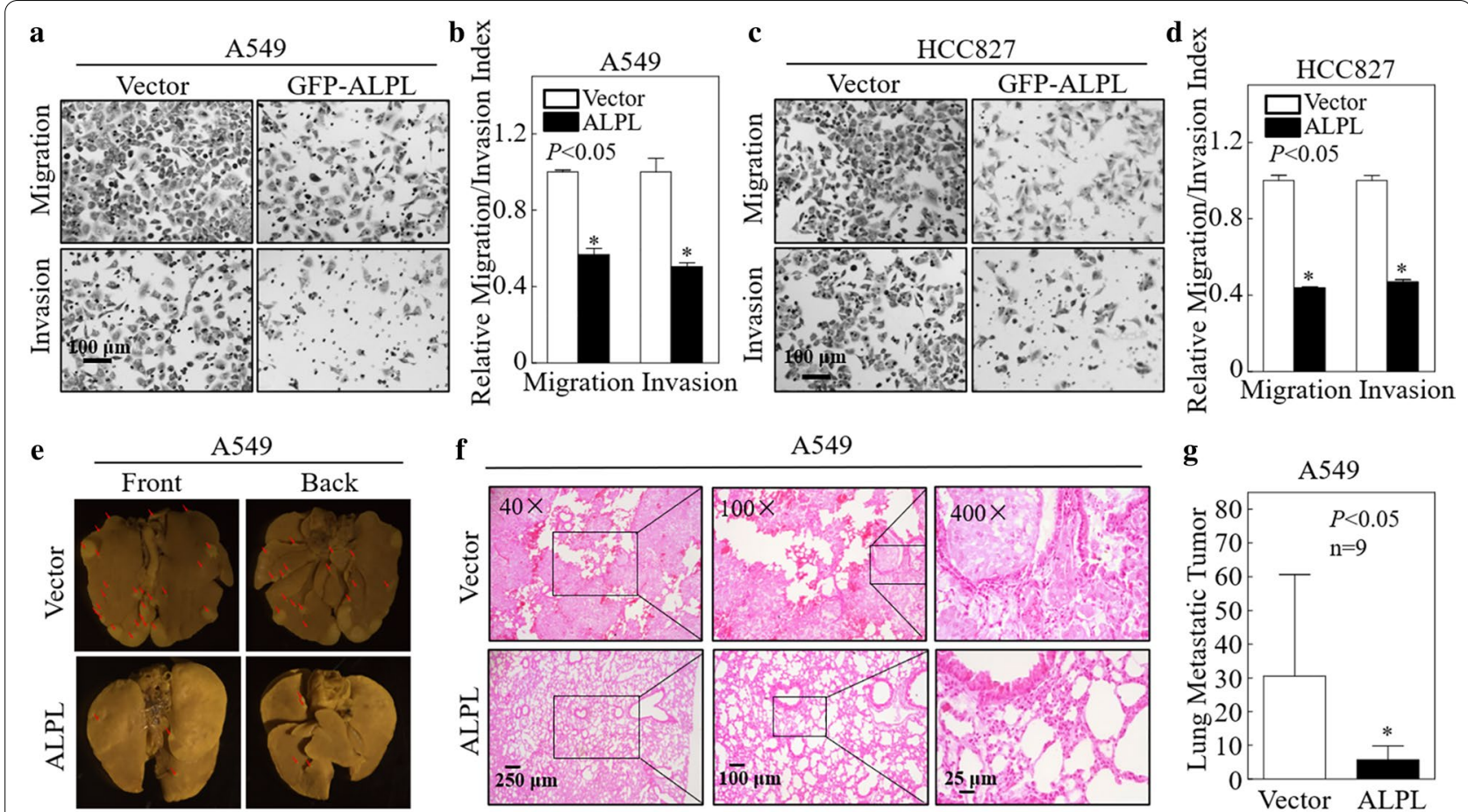

f

A549
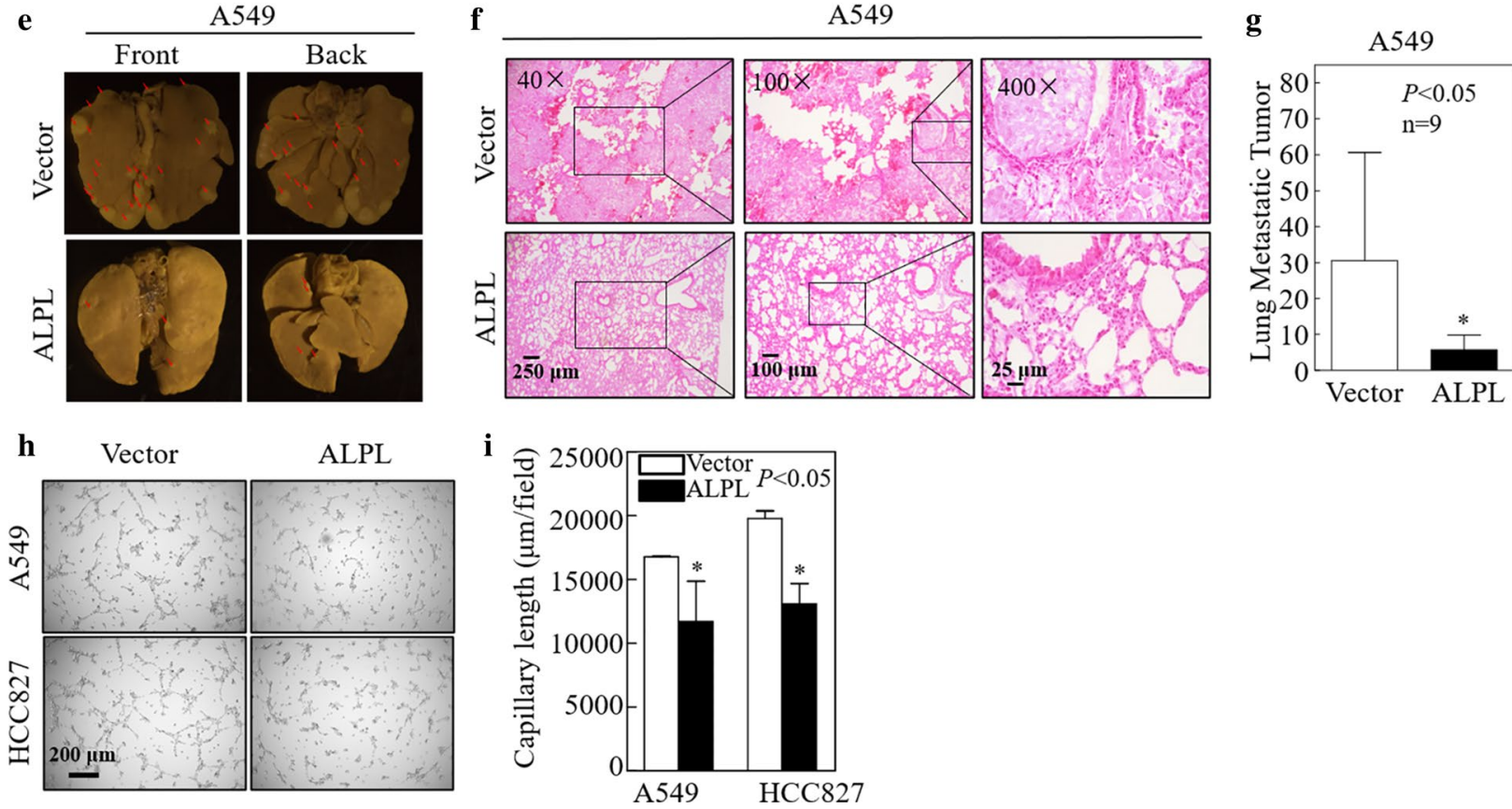

Fig. 3 Alkaline phosphatase (ALPL) inhibited the metastasis of LUAD cells in vivo and in vitro. Transwell assays were performed to determine the effect of ALPL overexpression on A549 (a) and HCC827 (c) cells, Scale bars: $100 \mu \mathrm{m}$. b, d The migration and invasion rate of A549 (a) and HCC827 (c) cells, $n=3$. e A tail vein injection model was used to evaluate lung metastasis. The front and back images of the lungs are shown, $n=9$. $\mathbf{f}$ Representative H\&E images of A549 (Vector) and A549 (ALPL) tumors, Scale bars: $250 \mu \mathrm{m}, 100 \mu \mathrm{m}$ or $25 \mu \mathrm{m}$. g Quantification of the number of metastatic lung tumors in five pairs of nude mice. $\mathbf{h}$ Tube formation assay were performed to determine the effect of ALPL overexpression on the angiogenesis activity of A549 and HCC827, Scale bars: $100 \mu \mathrm{m}$. i Capillary length was calculated in A549 and HCC827 cell culture medium. Data were presented as the mean \pm SD and analyzed by Student's $t$-test. Asterisks $\left(^{*}\right)$ represent statistical significance $(P<0.05)$

ALPL inhibits LUAD cell migration by downregulating RhoA To elucidate the molecular mechanism through which ALPL inhibits LUAD cell migration and invasion, western blot assays were performed to evaluate the expression of molecules related to cell motility. Matrix metalloproteinase (MMP)2 and MMP9 enhance the invasion of cancer cells by degrading type IV collagen [36, 37]. Slug, Snail, E-cadherin, $\mathrm{N}$-cadherin, and $\beta$-catenin, which are involved in epithelial-mesenchymal transition (EMT), have also been reported to promote cancer cell migration and invasion [17, 18, 38-43], while RhoA, Cdc42, and Rac1 are members of the Rho family of small GTPases, and are key mediators of actin polymerization and cell migration. These molecules play active roles in cell migration and invasion [22, 44, 45]. RhoA was consistently and significantly downregulated in A549 and HCC827 cells after overexpression of ALPL, while the other molecules were not consistently affected by ALPL expression (Fig. 4a). Therefore, we hypothesized that ALPL inhibited LUAD cell migration by downregulating RhoA expression. To validate this hypothesis, RhoA was stably overexpressed in the ALPL-overexpressing cell lines [A549 (ALPL) and HCC827 (ALPL)], as shown in Fig. 4b and e. Overexpression of RhoA restored the migratory 


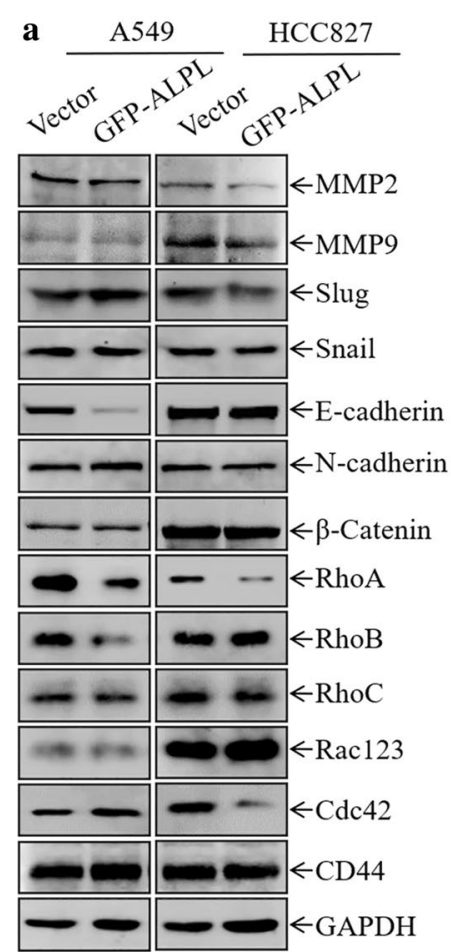

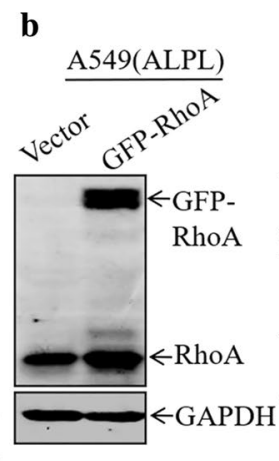

c

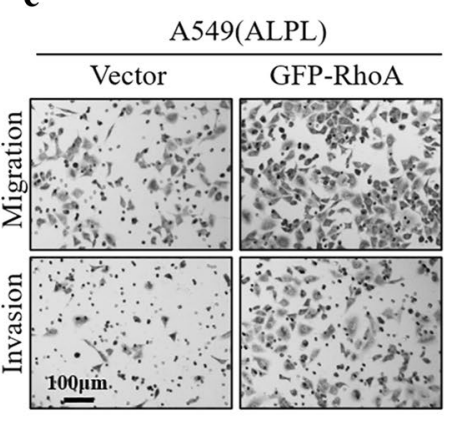

e $\operatorname{HCC} 827(A L P L)$

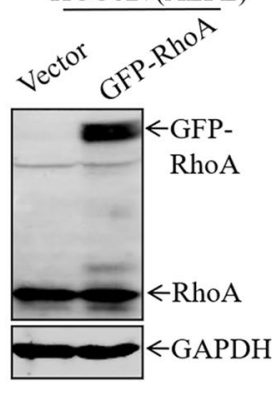

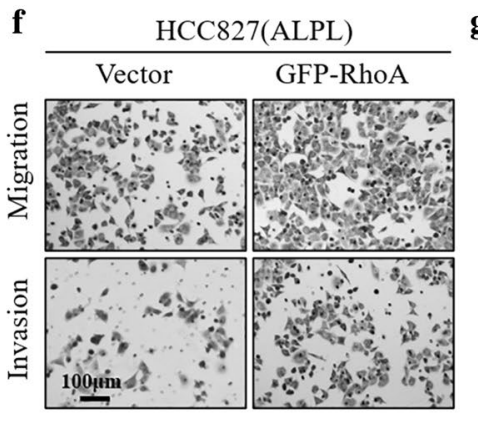

d
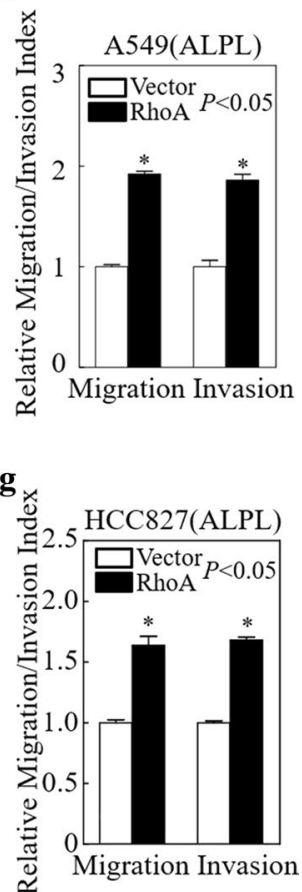

Fig. 4 Alkaline phosphatase (ALPL) inhibits the migration and invasion of LUAD cells by downregulating RhoA protein expression. a Western blot analysis of cell lysates from the indicated cells. b, e Western blot analysis of GFP-RhoA and RhoA expression in A549 (ALPL) and HCC827 (ALPL) cells stably transfected with GFP-RhoA or a vector control plasmid. GAPDH was used as a loading control. Transwell assays were performed to determine the effect of RhoA overexpression on A549 (ALPL) (c) and HCC827 (ALPL) (f) cells, Scale bars: $100 \mu \mathrm{m}$. d, g The migration and invasion rate of A549 (ALPL) and HCC827 (ALPL) cells. Data were presented as the mean \pm SD and analyzed by Student's $t$-test, $n=3$. Asterisks $\left({ }^{*}\right.$ ) indicate a significant increase relative to control vector cells $(P<0.05)$

ability of A549 and HCC827 transfectants (Fig. 4c, d and $\mathrm{f}, \mathrm{g})$. These findings suggest that ALPL inhibits LUAD cell migration by downregulating RhoA.

ALPL inhibits RhoA transcription by downregulating c-Myc To further explore the mechanism by which ALPL downregulates RhoA expression, we first conducted a semiquantitative PCR analysis of the RhoA mRNA level. RhoA mRNA was significantly downregulated in A549 (ALPL) and HCC827 (ALPL) cells by PCR (Fig. 5a) and qPCR (Fig. 5b). These data indicated that ALPL downregulated RhoA expression at the mRNA level. To investigate the mechanism underlying ALPL downregulation of RhoA, we investigated $R h o A$ promoter activity by dual-luciferase reporter assays. As shown in Fig. 5c, RhoA promoter activity was significantly downregulated in both cell lines after ALPL overexpression, indicating that ALPL downregulates RhoA expression by inhibiting its transcription.

To further investigate the effects of ALPL on RhoA transcription, the TFANSFAC Transcription Factor Binding Sites Software (Biological Database, Wolfenbuttel, Germany) was used to predict transcription factors controlling RhoA expression. Transcription factors for which potentially conserved binding sites in the RhoA promoter region were identified included c-Myc, c-Jun, Foxp3, and Sp1 (Fig. 5d). We examined the expression of these potential transcription factors in A549 and HCC 827 cells by western blot analysis. c-Myc protein levels were significantly downregulated in A549 (ALPL) and HCC827 (ALPL) cells, while the other molecules showed no signs of consistent downregulation (Fig. 5e). It has been reported that c-Myc directly regulates $R h o A$ transcription [44]. We hypothesized that c-Myc may be a key molecule in the mechanism through which ALPL regulates $R h o A$ transcription. To test this hypothesis, we constructed an A549 (ALPL) stable cell line that expresses c-Myc and found that RhoA protein expression was restored (Fig. 5f). Subsequent qPCR analysis confirmed that RhoA expression was also restored at the mRNA level (Fig. 5g). Dual-luciferase reporter assays also showed that overexpression of c-Myc restored the level of RhoA promoter activity in A549 (ALPL) cells (Fig. 5h). These data suggested that ALPL-downregulated c-Myc expression in LUAD resulted in reduced RhoA expression, which in turn resulted in decreased LUAD cell 
a
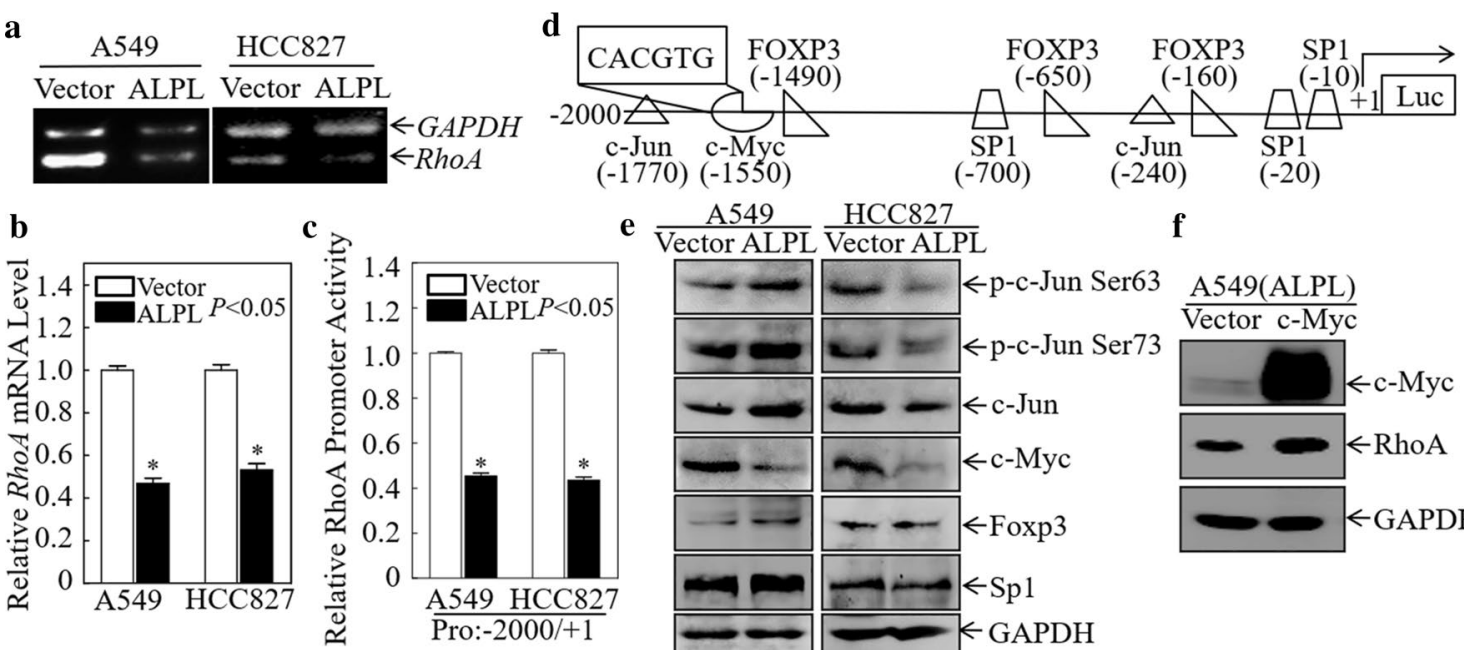

e

$\frac{\text { A549 }}{\text { Vector ALPL }} \frac{\text { HCC827 }}{\text { Vector ALPL }}$ $(-700)$ $(-240)$ $(-20)$

\section{f}
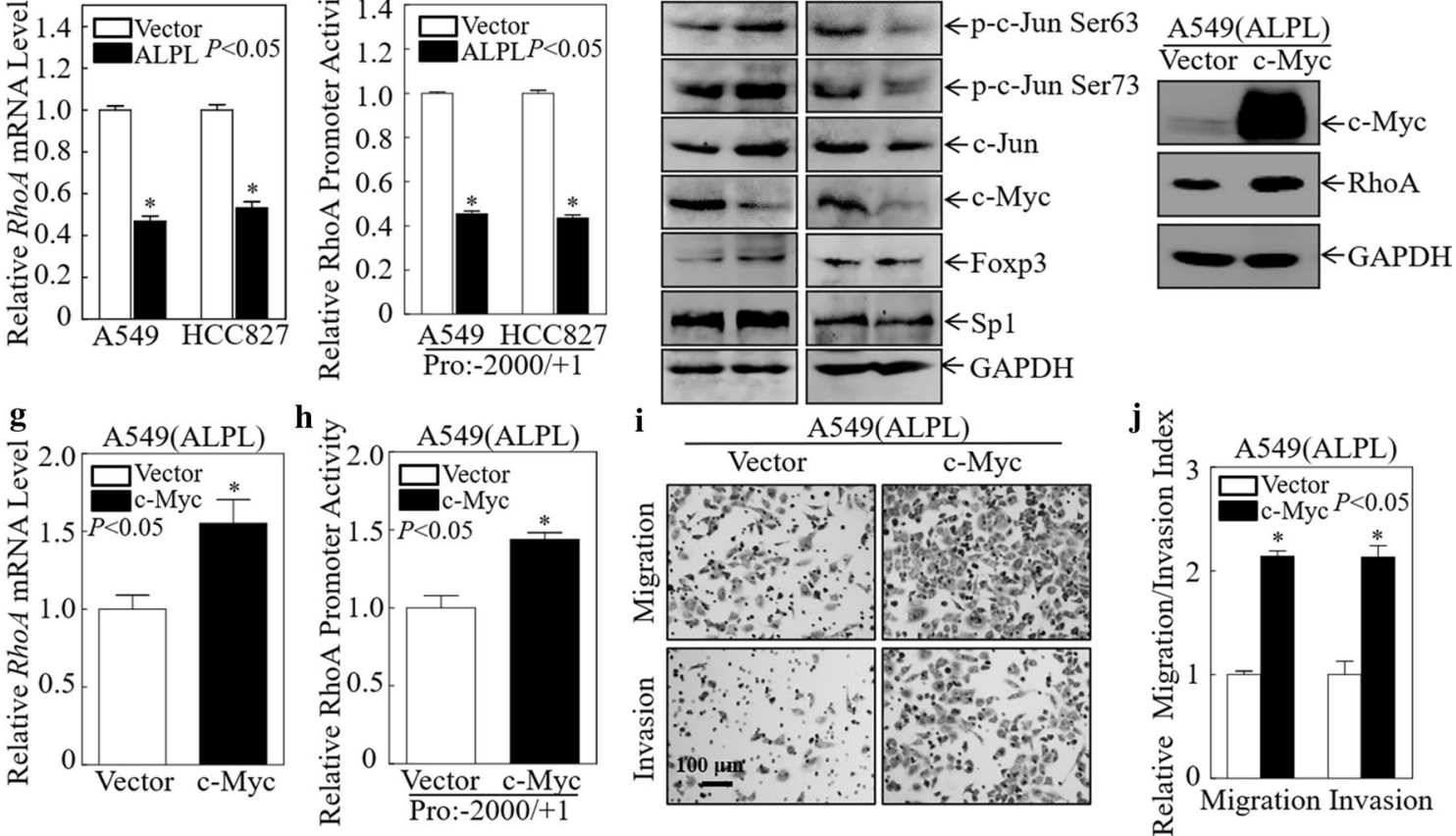

Fig. 5 Alkaline phosphatase (ALPL) inhibits RhoA mRNA transcription by downregulating c-Myc. a Semi-quantitative PCR was used to evaluate RhoA mRNA expression after ALPL overexpression in A549 and HCC827 cells. b RhoA mRNA expression was evaluated by qPCR after ALPL overexpression in A549 and HCC827 cells. c Dual-luciferase reporter assays were used to detect RhoA promoter activity after ALPL overexpression in A549 and HCC827 cells. d TFANSFAC Transcription Factor Binding Sites Software (Biological Database, Wolfenbuttel, Germany) was used for bioinformatics analysis of the RhoA promoter region. Transcription factors for which potentially conserved binding sites were identified in the RhoA promoter region included c-Myc, c-Jun, Foxp3, and Sp1. e Western blot analysis of cell lysates from the indicated cells. GAPDH was used as an internal control. f Western blot analysis of c-Myc and RhoA expression in A549 (ALPL) cells stably transfected with c-Myc or a control plasmid. GAPDH was used as a loading control. $\mathbf{g}$ The RhoA mRNA expression level was evaluated by qPCR after overexpression of c-Myc in A549 (ALPL) cells. h Dual-luciferase reporter assays of the RhoA promoter activity after overexpression of c-Myc in A549 (ALPL) cells. i Transwell assays to determine the effect of overexpression of c-Myc on A549 (ALPL) cells, Scale bars: $100 \mu \mathrm{m}$. $\mathbf{j}$ The migration and invasion rate of A549 (ALPL) cells. All results were presented as the mean \pm SD and analyzed by Student's $t$-test, $n=3$. Asterisks $\left(^{*}\right)$ represent statistical significance $(P<0.05)$

migration and invasion. To further verify this, we performed three independent Transwell experiments following transfection of A549 (ALPL) cells with c-Myc. The migration and invasion ability of A549 (ALPL) cells was restored following transfection with c-Myc (Fig. 5i, j). These data confirm that ALPL downregulates RhoA by inhibiting the expression of c-Myc, resulting in reduced LUAD cell migration and invasion.

ALPL promotes the degradation of c-Myc by activating ERK To further explore the mechanism by which ALPL regulates c-Myc, we analyzed c-Myc mRNA levels by qPCR. There were no significant differences in c-Myc mRNA levels in A549 (ALPL) and HCC827 (ALPL) cells compared with the corresponding wild-type cell lines (Fig. 6a), indicating that ALPL does not regulate $\mathrm{c}-\mathrm{Myc}$ at the transcriptional level. A variety of proteins have been reported to play roles in cancer development by regulating the degradation of c-Myc protein $[45,46]$. Therefore, we evaluated c-Myc protein degradation in control A549 and A549 (ALPL) cells. ALPL overexpression significantly enhanced the degradation rate of c-Myc protein (Fig. 6b), indicating that ALPL downregulates $\mathrm{c}-\mathrm{Myc}$ expression by enhancing its degradation. It has been reported that degradation of c-Myc usually occurs via classical ubiquitin-dependent degradation pathways, in which ERK- and GSK-3-mediated phosphorylation of c-Myc at Ser62 and Thr58 tags it 
$\mathbf{a}$

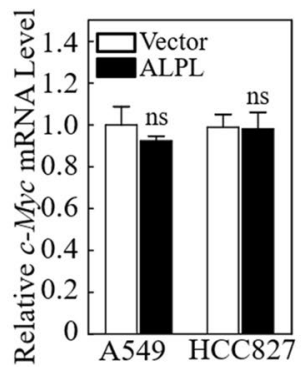

c

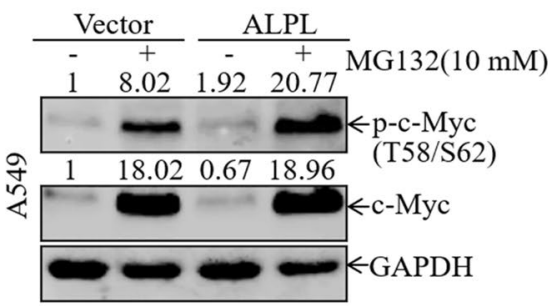

d b
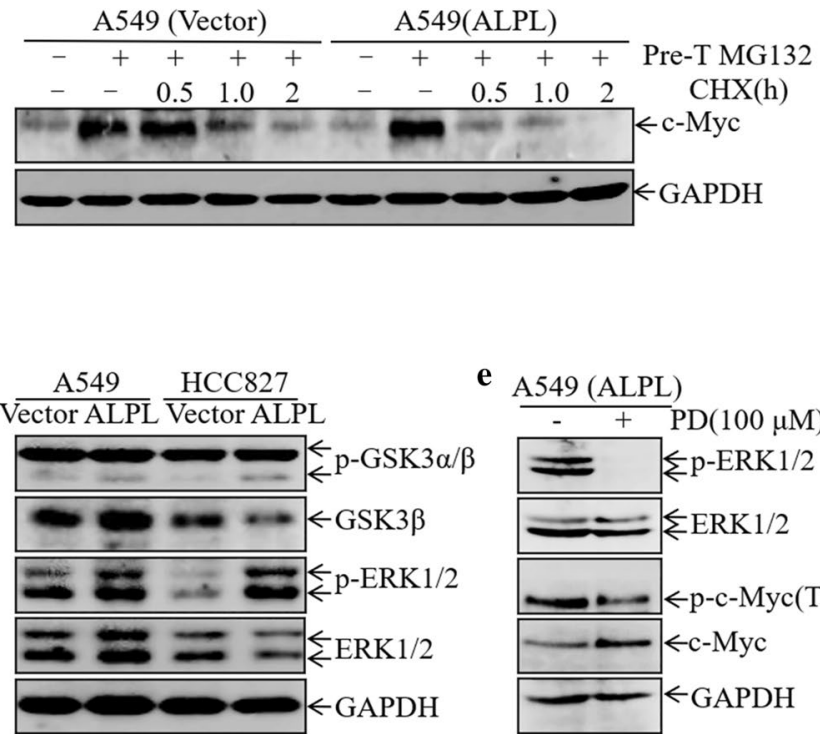

e A549 (ALPL)

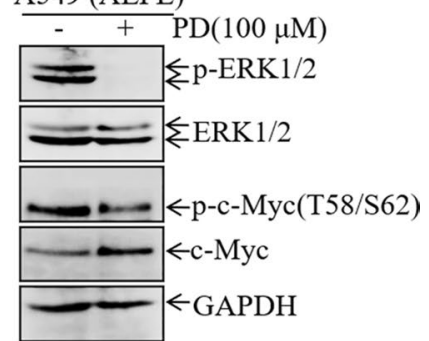

Fig. 6 Alkaline phosphatase (ALPL) promotes the degradation of c-Myc by activating ERK. a qPCR analysis of the levels of c-Myc mRNA in A549 and HCC827 cells overexpressing ALPL. ns, not significant. b The rate of c-Myc protein degradation in A549 (Vector) and A549 (ALPL) cells. GAPDH was used as an internal control. c Western blot analysis of the levels of c-Myc and p-c-Myc (Thr58/Ser62) in A549 (Vector) and A549 (ALPL) cells after treatment with MG132. $\mathbf{d}$ Western blot analysis of the indicated cells for levels of the ubiquitin-related molecules ERK1/2, GSK-3 $\beta, p-E R K 1 / 2$, and p-GSK-3a/ $\beta$. GAPDH was used as an internal control. e Western blot analysis of the levels of p-ERK1/2, ERK1/2, c-Myc, and p-c-Myc (Thr58/Ser62) in A549 (ALPL) cells after treatment with PD98059 (100 $\mu \mathrm{M})$

for degradation [47, 48]. To confirm that ALPL degradation of c-Myc is ubiquitin-dependent, we investigated the effects of the proteasome inhibitor MG132 on c-Myc levels and phosphorylation in control A549 and A549 (ALPL) cells. After treatment with MG132, c-Myc accumulated in both the control A549 and A549 (ALPL) cells, with no significant difference between the two cell lines. However, levels of p-c-Myc (Thr58/ Ser62) were much higher in A549 (ALPL) cells than in control cells (Fig. 6c). These results suggested that ALPL accelerated the degradation of c-Myc by promoting its phosphorylation at Thr58 and Ser62, resulting in ubiquitin-dependent degradation.

To determine whether c-Myc phosphorylation at Thr58 and Ser62 was mediated by ERK or GSK-3, we analyzed the expression of ERK1/2, GSK-3 $\beta$, p-ERK $1 / 2$, and p-GSK-3 $\alpha / \beta$ (Ser21/9) in both A549 (ALPL) and HCC827 (ALPL) cells (Fig. 6d). Western blot analysis showed that p-ERK and p-GSK-3 $\beta$ (Ser9) were significantly upregulated in A549 (ALPL) and HCC827 (ALPL) cells, while the other molecules were not significantly affected by ALPL overexpression. GSK-3 activity is significantly inhibited after phosphorylation at Ser9; therefore, we further speculated that ALPL accelerated the degradation of c-Myc by activating ERK1/2, resulting in the increase in p-c-Myc (Thr58/Ser62). To test this hypothesis, A549
(ALPL) cells were treated with the p-ERK inhibitor PD-98059. Phosphorylation of ERK was almost completely inhibited in the PD-98059-treated cells, while c-Myc expression increased and the p-c-Myc (Thr58/ Ser62) level decreased (Fig. 6e). These findings confirmed our hypothesis that ALPL promotes phosphorylation of c-Myc by activating ERK, leading to accelerated c-Myc degradation.

\section{ALPL overexpression increases p-ERK accompanied with c-Myc and RhoA downregulation in metastatic lung tumor tissues of mouse model}

To determine whether ALPL exerts a regulatory effect on $\mathrm{p}-\mathrm{ERK} / \mathrm{c}-\mathrm{Myc} / \mathrm{RhoA}$ in vivo, we assessed the expression of ALPL, p-ERK, c-Myc and RhoA using IHC in metastatic lung tumor tissues of mouse models that are generated with A549 (ALPL) and HCC827 (ALPL) in comparison to A549 (Vector) and HCC827 (Vector), respectively, and found that a remarkable upregulation of p-ERK but c-Myc and RhoA downregulation in metastatic lung tumor tissues of mouse models generated by injection of A549 (ALPL) and HCC827(ALPL) cells (Fig. 7a-d, Additional file 1: Fig. S8a-d). RhoA is positively correlated with c-Myc expression, while c-Myc expression is negatively correlated with p-ERK expression. Collectively, these results demonstrated that ALPL 


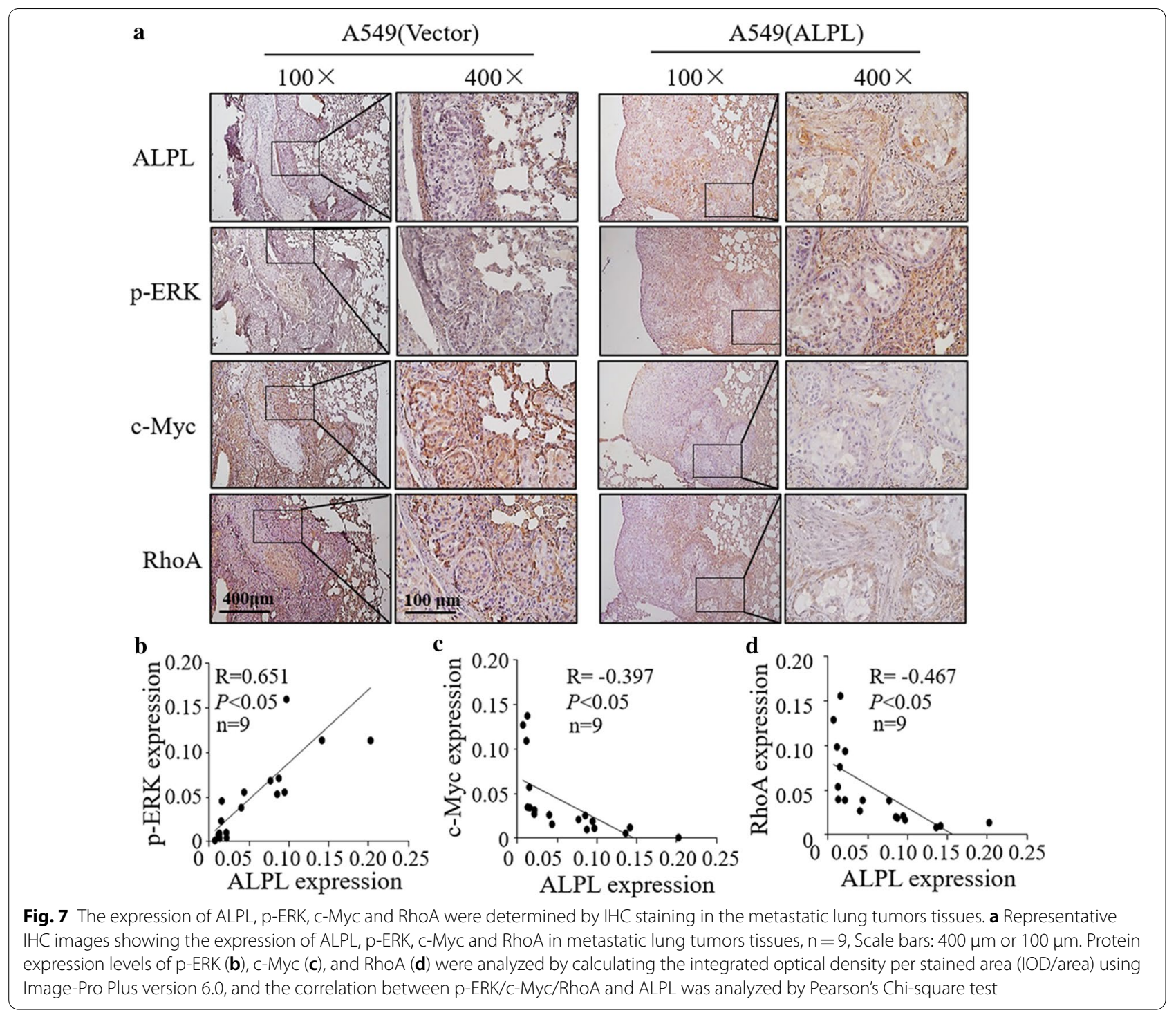

expression downregulated c-Myc by enhancing ERK phosphorylation, leading to a decrease in c-Myc-mediated RhoA protein expression, which eventually suppresses LUAD cell metastasis (Fig. 8).

\section{Discussion}

In this study, we show for the first time that ALPL specially inhibits the metastasis of LUAD cells but not LUSC cells. This not only indicates that ALPL plays a unique role in the pathological development of LUAD, but also implicates ALPL as a specific target for inhibiting the metastasis of LUAD. We also show that the inhibitory effects of ALPL on the metastasis of LUAD cells are mediated via the $\mathrm{p}-\mathrm{ERK} / \mathrm{c}-\mathrm{Myc} / \mathrm{RhoA}$ axis. ERK, c-Myc and RhoA are the genes playing vital roles in tumor metastasis of many cancers including lung cancer. It is of great significance to develop drugs targeting ERK, $\mathrm{c}-\mathrm{Myc}$ and RhoA for the treatment and prevention of tumor metastasis. Our results showed that ALPL can significantly inhibit the invasion of LUAD cells in vitro and in vivo, which is mediated via the ALPL/p-ERK/c-Myc/ RhoA axis. Therefore, our findings provide a theoretical basis for the development of strategies targeting ALPL for the treatment of LUAD.

ALPL is commonly used to evaluate acute liver damage and cholestasis [49]. Studies have also shown that ALPL is associated with hypophosphatemia [5, 50], skeletal development, cardiovascular disease, and acute kidney injury [51-53]. It has been reported that mutations in ALPL affect its ability to cause disease [5]. We did not identify any mutations in ALPL in LUAD using the TCGA database, suggesting that metastasis of LUAD 


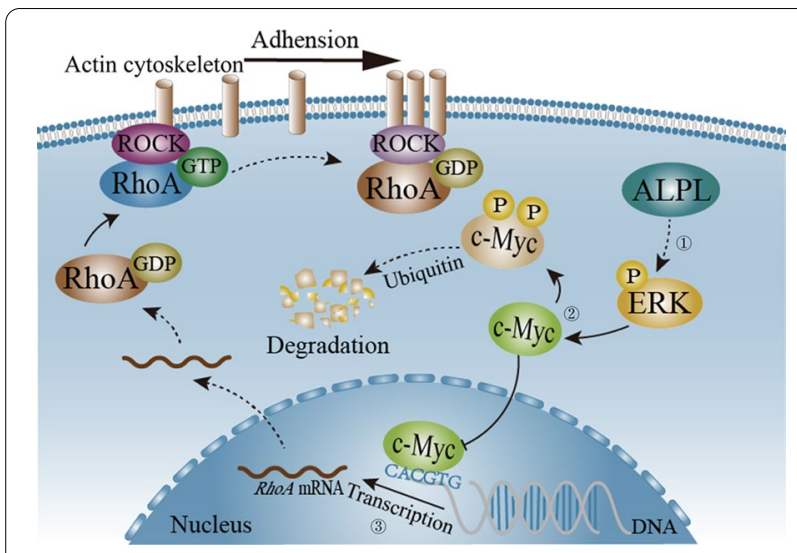

Fig. 8 Schematic diagram of the pathway by which ALPL downregulates RhoA via c-Myc. (1) ALPL leads to an accumulation of phosphorylated (p)-ERK. (2) p-ERK promotes c-Myc phosphorylation at Thr58 and Ser62 which is then degradated through ubiquitinproteasome system. (3) The reduction of c-Myc leads to a decrease in RhoA transcription, which eventually suppresses LUAD cell metastasis. The dotted line indicates a multi-process, and the solid line indicates a single process

cells is related to inhibition of ALPL expression rather than mutation. LUAD cells also had lower expression of ALPL than normal lung cells. ALPL also plays very different roles in different tumor types. Reduced expression of tumor-derived ALPL inhibited mesenchymal-to-epithelial transition and the migration of advanced prostate cancer [10]. Conversely, ALPL functions as a tumor suppressor gene in meningioma [54] and ovarian cancer [13]. In this study, we show for the first time that ALPL inhibits the metastasis of LUAD cells via the p-ERK1/2-c-MycRhoA axis, which extends our understanding of the role of ALPL in cancer development. Although the expression of ALPL is not tissue-specific, the function of ALPL is influenced by its cellular context. ALPL expression is elevated after acute injury of some organs such as the liver and kidneys $[49,53]$. Our finding that downregulation of ALPL promotes metastasis of LUAD cells highlights the diverse functions of ALPL in different settings. We further found that ALPL inhibited metastasis of LUAD cells but did not play a similar role in LUSC cells, and this phenomenon deserves further exploration.

In this study, we demonstrated that ALPL enhances ERK phosphorylation; however, previous studies have shown that ALPL downregulates its targets [6]. Lee et al. found that the human dental pulp cells were cultured in osteogenic medium, ALPL and p-ERK were up-regulated, while MKP-1 was downregulated [55]. As a member of the MAP kinase phosphatase (MKP) family, MKP-1 inactivates MAP kinases including ERK1/2 [56], and its degradation can be attenuated by inhibitors of the ubiquitin-directed proteasome complex. We speculate that
ALPL may increase ERK phosphorylation by regulating MKP-1, although this remains to be clarified. ERK is a member of the MAPK family and promotes ubiquitindependent degradation of c-Myc by phosphorylation [47, 48]. In our study, ALPL increased ERK phosphorylation, resulting in increased phosphorylation of c-Myc, thereby promoting c-Myc degradation. Furthermore, in LUAD cells, c-Myc degradation was reduced because p-ERK was reduced. Previous studies have shown that GSK3 plays a key role in the ubiquitin-dependent degradation of c-Myc $[47,48]$. However, our results indicated that p-ERK, not p-GSK3, is the key molecule through which ALPL promotes c-Myc ubiquitination. This finding led us to reexamine the status of p-ERK in the ubiquitin-dependent degradation of c-Myc.

c-Myc promotes RhoA mRNA expression, thereby enhancing the migration and invasion ability of tumor cells [44]. We demonstrate here that ALPL enhances the ubiquitin-dependent degradation of c-Myc by p-ERK. c-Myc is a well-known oncogene [15], and inhibition of c-Myc in cancer is a potential therapeutic strategy. Thus, the ability of ALPL to down-regulate c-Myc may represent a novel approach to cancer treatment. As a member of the Rho family, RhoA plays an important role in cancer cell metastasis [23, 24]. Overexpression of RhoA significantly restored the inhibitory effect of ALPL on LUAD cells metastasis. Our study is the first to show that ALPL reduces the expression of RhoA to inhibit the metastasis of LUAD cells. In summary, ALPL promotes ubiquitin-dependent c-Myc degradation by activating ERK. Reduced c-Myc expression results in reduced RhoA transcription, thereby inhibiting cell migration and invasion. Accordingly, downregulation of ALPL decreased ERK phosphorylation in LUAD, causing an accumulation of c-Myc, which promotes RhoA transcription, leading to increased metastasis of LUAD cells (Fig. 8).

\section{Conclusions}

Overall, we evidenced for the first time that ALPL specially inhibits the metastasis of LUAD cells but not LUSC cells, and revealed a molecular mechanism through which ALPL regulates LUAD invasion and metastasis by affecting the $\mathrm{p}-\mathrm{ERK} / \mathrm{c}-\mathrm{Myc} / \mathrm{RhoA}$ axis. These results implicated ALPL as a specific target for inhibiting the metastasis of LUAD and provided a theoretical basis for the targeted therapy of clinical LUAD.

\section{Supplementary Information}

The online version contains supplementary material available at https://doi. org/10.1186/s12935-021-01919-7.

Additional file 1.Additional Table and Figures. 


\section{Acknowledgements}

We thank participants, specimen donors and research groups who participated in the data set of the TCGA lung cancer data set to contribute to the database construction.

\section{Authors' contributions}

$\mathrm{LZ}, \mathrm{HH}$ and $\mathrm{XH}$ conceived and designed the study; ZL, WL, HZ and LL detected the cells' biological function, performed the qPCR assays, carried out the soft agar and luciferase reporter assays, and conducted the statistical analyses. $\mathrm{HZ}$ and WL performed the animal experiments and the $\mathrm{IHC}$ staining assays. $\mathrm{HZ}, \mathrm{LL}$ and QX collected clinical samples. $\mathrm{HH}, \mathrm{WL}, \mathrm{XH}$ and $\mathrm{LZ}$ drafted the manuscript. All authors read and approved the final version of the manuscript.

\section{Funding}

This work was supported by the National Natural Science Foundation of China (NSFC81872587); Wenzhou Medical University (89219018 and 89219042); Key Project of Science and Technology Innovation Team of Zhejiang Province (2013TD10) and Key Discipline of Zhejiang Province in Medical Technology (First Class, Category A).

\section{Availability of data and materials}

Bioinformatics results announced here based on data produced by the TCGA research network (http://cancergenome.nih.gov/). All data generated or analyzed during this study are included in this published article and its supplementary information files.

\section{Declarations}

\section{Ethics approval and consent to participate}

The Ethics Committee of Wenzhou Medical University approved this study (\#2019-086). Animal experiments were performed at the Animal Institute of Wenzhou Medical University according to the protocols approved by the Laboratory Animal Center of Wenzhou Medical University and the Laboratory Animal Ethics Committee of Wenzhou Medical University.

\section{Consent for publication}

Not applicable.

\section{Competing interests}

The authors declare that they have no competing interests.

\section{Author details}

${ }^{1}$ Zhejiang Provincial Key Laboratory of Medical Genetics, Key Laboratory of Laboratory Medicine, Ministry of Education, School of Laboratory Medicine and Life Sciences, Wenzhou Medical University, Wenzhou 325035, Zhejiang, China. ${ }^{2}$ The First Affiliated Hospital of Wenzhou Medical University, Wenzhou 325035, Zhejiang, China. ${ }^{3}$ Zhejiang Provincial Key Laboratory of Pancreatic Disease, School of Medicine, the First Affiliated Hospital, Zhejiang University, Hangzhou 310003, Zhejiang, China.

Received: 25 December 2020 Accepted: 7 April 2021 Published online: 15 April 2021

\section{References}

1. Sung H, Ferlay J, Siegel RL, Laversanne M, Soerjomataram I, Jemal A, et al. Global cancer statistics 2020: GLOBOCAN estimates of incidence and mortality worldwide for 36 cancers in 185 countries. Cancer J Clin. 2021. https://doi.org/10.3322/caac.21660.

2. Travis WD, Brambilla E, Nicholson AG, Yatabe Y, Austin JHM, Beasley MB, et al. The 2015 World Health Organization classification of lung tumors: impact of genetic, clinical and radiologic advances since the 2004 classification. J Thorac Oncol. 2015;10:1243-60.

3. Osmani L, Askin F, Gabrielson E, Li QK. Current WHO guidelines and the critical role of immunohistochemical markers in the subclassification of non-small cell lung carcinoma (NSCLC): Moving from targeted therapy to immunotherapy. Semin Cancer Biol. 2018;52:103-9.

4. Siegel RL, Miller KD, Jemal A. Cancer statistics, 2018. CA Cancer J Clin. 2018:68:7-30
5. Whyte MP. Hypophosphatasia - aetiology, nosology, pathogenesis, diagnosis and treatment. Nat Rev Endocrinol. 2016;12:233-46.

6. Zheng F, Guo S, Zeng F, Li J, Wu S. Ratiometric fluorescent probe for alkaline phosphatase based on betaine-modified polyethylenimine via excimer/monomer conversion. Anal Chem. 2014;86:9873-9.

7. Lin L, Liu Y, Yan J, Wang X, Li J. Sensitive nanochannel biosensor for T4 polynucleotide kinase activity and inhibition detection. Anal Chem. 2013;85:334-40.

8. Wang Z, Sun N, He Y, Liu Y, Li J. DNA assembled gold nanoparticles polymeric network blocks modular highly sensitive electrochemical biosensors for protein kinase activity analysis and inhibition. Anal Chem. 2014;86:6153-9.

9. Xiao T, Sun J, Zhao J, Wang S, Liu G, Yang X. FRET effect between fluorescent polydopamine nanoparticles and $\mathrm{MnO} 2$ nanosheets and its application for sensitive sensing of alkaline phosphatase. ACS Appl Mater Interfaces. 2018;10:6560-9.

10. Rao SR, Snaith AE, Marino D, Cheng X, Lwin ST, Orriss IR, et al. Tumourderived alkaline phosphatase regulates tumour growth, epithelial plasticity and disease-free survival in metastatic prostate cancer. Br J Cancer. 2017;116:227-36.

11. Chen SC, Tsai SP, Jhao JY, Jiang WK, Tsao CK, Chang LY. Liver fat, hepatic enzymes, alkaline phosphatase and the risk of incident type 2 diabetes: a prospective study of 132,377 adults. Sci Rep. 2017;7:4649.

12. Brichacek AL, Brown CM. Alkaline phosphatase: a potential biomarker for stroke and implications for treatment. Metab Brain Dis. 2019;34:3-19.

13. Luo M, Zhou L, Zhan SJ, Cheng LJ, Li RN, Wang H, et al. ALPL regulates the aggressive potential of high grade serous ovarian cancer cells via a non-canonical WNT pathway. Biochem Biophys Res Commun. 2019:513:528-33.

14. Dalla-Favera R, Bregni M, Erikson J, Patterson D, Gallo RC, Croce CM. Human c-myc onc gene is located on the region of chromosome 8 that is translocated in Burkitt lymphoma cells. Proc Natl Acad Sci USA. 1982;79:7824-7.

15. Taub R, Kirsch I, Morton C, Lenoir G, Swan D, Tronick S, et al. Translocation of the c-myc gene into the immunoglobulin heavy chain locus in human Burkitt lymphoma and murine plasmacytoma cells. Proc Natl Acad Sci USA. 1982;79:7837-41.

16. Zack TI, Schumacher SE, Carter SL, Cherniack AD, Saksena G, Tabak $B$, et al. Pan-cancer patterns of somatic copy number alteration. Nat Genet. 2013:45:1134-40.

17. Gottardi CJ, Wong E, Gumbiner BM. E-cadherin suppresses cellular transformation by inhibiting beta-catenin signaling in an adhesionindependent manner. J Cell Biol. 2001;153:1049-60.

18. Chen HN, Yuan K, Xie N, Wang K, Huang Z, Chen Y, et al. PDLIM1 stabilizes the E-Cadherin/beta-Catenin complex to prevent epithelialmesenchymal transition and metastatic potential of colorectal cancer cells. Cancer Res. 2016;76:1122-34.

19. Cau J, Hall A. Cdc42 controls the polarity of the actin and microtubule cytoskeletons through two distinct signal transduction pathways. J Cell Sci. 2005;118:2579-87.

20. Jaffe AB, Hall A. Rho GTPases: biochemistry and biology. Annu Rev Cell Dev Biol. 2005;21:247-69.

21. Burridge K, Wennerberg K. Rho and Rac take center stage. Cell. 2004;116:167-79.

22. Narumiya S, Tanji M, Ishizaki T. Rho signaling, ROCK and mDia1, in transformation, metastasis and invasion. Cancer Metastasis Rev. 2009;28:65-76.

23. Andreux PA, Williams EG, Koutnikova H, Houtkooper $\mathrm{RH}$, Champy $M F$, Henry $H$, et al. Systems genetics of metabolism: the use of the BXD murine reference panel for multiscalar integration of traits. Cell. 2012;150:1287-99.

24. Galluzzi L, Vitale I, Senovilla L, Olaussen KA, Pinna G, Eisenberg T, et al. Prognostic impact of vitamin B6 metabolism in lung cancer. Cell Rep. 2012:2:257-69.

25. Li X, Tian Z, Jin H, Xu J, Hua X, Yan H, et al. Decreased c-Myc mRNA stability via the microRNA 141-3p/AUF1 axis is crucial for p63alpha inhibition of cyclin D1 gene transcription and bladder cancer cell tumorigenicity. Mol Cell Biol. 2018;38:e00273.

26. Jin $\mathrm{H}$, Sun $W$, Zhang $Y$, Yan $H$, Liufu H, Wang S, et al. MicroRNA-411 downregulation enhances tumor growth by upregulating MLLT11 
expression in human bladder cancer. Mol Ther Nucleic Acids. 2018;11:312-22.

27. Jin $H$, Xue L, Mo L, Zhang D, Guo X, Xu J, et al. Downregulation of miR-200c stabilizes XIAP mRNA and contributes to invasion and lung metastasis of bladder cancer. Cell Adh Migr. 2019;13:236-48.

28. Du K, Yu Y, Zhang D, Luo W, Huang H, Chen J, et al. NFkappaB1 (p50) suppresses SOD2 expression by inhibiting FoxO3a transactivation in a miR1 90/PHLPP1/Akt-dependent axis. Mol Biol Cell. 2013;24:3577-83.

29. Zhu J, Tian Z, Li Y, Hua X, Zhang D, Li J, et al. ATG7 Promotes bladder cancer invasion via autophagy-mediated increased ARHGDIB mRNA stability. Adv Sci (Weinh). 2019;6:1801927.

30. Huang H, Zhu J, Li Y, Zhang L, Gu J, Xie Q, et al. Upregulation of SQSTM1/ p62 contributes to nickel-induced malignant transformation of human bronchial epithelial cells. Autophagy. 2016;12:1687-703.

31. Zeng X, Xu Z, Gu J, Huang H, Gao G, Zhang X, et al. Induction of miR-137 by isorhapontigenin (ISO) directly targets Sp1 protein translation and mediates its anticancer activity both in vitro and in vivo. Mol Cancer Ther. 2016;15:512-22.

32. Zhang $D$, Wang $Y$, Liang $Y$, Zhang $M$, Wei J, Zheng $X$, et al. Loss of $p 27$ upregulates MnSOD in a STAT3-dependent manner, disrupts intracellular redox activity and enhances cell migration. J Cell Sci. 2014;127:2920-33.

33. Sui H, Cai GX, Pan SF, Deng WL, Wang YW, Chen ZS, et al. miR200c attenuates P-gp-mediated MDR and metastasis by targeting JNK2/C-Jun signaling pathway in colorectal cancer. Mol Cancer Ther. 2014;13:3137-51.

34. Wei J, Du K, Cai Q, Ma L, Jiao Z, Tan J, et al. Lead induces COX-2 expression in glial cells in a NFAT-dependent, AP-1/NFkappaB-independent manner. Toxicology. 2014;325:67-73.

35. Sun W, Li S, Yu Y, Jin H, Xie Q, Hua X, et al. MicroRNA-3648 is upregulated to suppress TCF21, resulting in promotion of invasion and metastasis of human bladder cancer. Mol Ther Nucleic Acids. 2019;16:519-30.

36. Nagase $H$, Visse $R$, Murphy $G$. Structure and function of matrix metalloproteinases and TIMPs. Cardiovasc Res. 2006;69:562-73.

37. Zhao H, Achreja A, lessi E, Logozzi M, Mizzoni D, Di Raimo R, et al. The key role of extracellular vesicles in the metastatic process. Biochim Biophys Acta Rev Cancer. 2018;1869:64-77.

38. Kudo-Saito C, Shirako H, Takeuchi T, Kawakami Y. Cancer metastasis is accelerated through immunosuppression during Snail-induced EMT of cancer cells. Cancer Cell. 2009:15:195-206.

39. Hajra KM, Chen DY, Fearon ER. The SLUG zinc-finger protein represses E-cadherin in breast cancer. Cancer Res. 2002;62:1613-8.

40. Martin TA, Goyal A, Watkins G, Jiang WG. Expression of the transcription factors snail, slug, and twist and their clinical significance in human breast cancer. Ann Surg Oncol. 2005;12:488-96.

41. Uygur B, WU WS. SLUG promotes prostate cancer cell migration and invasion via CXCR4/CXCL12 axis. Mol Cancer. 2011;10:139.

42. Ahmed N, Thompson EW, Quinn MA. Epithelial-mesenchymal interconversions in normal ovarian surface epithelium and ovarian carcinomas: an exception to the norm. J Cell Physiol. 2007;213:581-8.
43. Morali OG, Delmas V, Moore R, Jeanney C, Thiery JP, Larue L. IGF-II induces rapid beta-catenin relocation to the nucleus during epithelium to mesenchyme transition. Oncogene. 2001;20:4942-50.

44. Pertz O, Hodgson L, Klemke RL, Hahn KM. Spatiotemporal dynamics of RhoA activity in migrating cells. Nature. 2006;440:1069-72.

45. Yeh E, Cunningham M, Arnold H, Chasse D, Monteith T, Ivaldi G, et al. A signalling pathway controlling C-Myc degradation that impacts oncogenic transformation of human cells. Nat Cell Biol. 2004;6:308-18.

46. Jain S, Wang $X$, Chang CC, Ibarra-Drendall C, Wang H, Zhang Q, et al. Src Inhibition Blocks c-Myc Translation and Glucose Metabolism to Prevent the Development of Breast Cancer. Cancer Res. 2015;75:4863-75.

47. Sears R, Nuckolls F, Haura E, Taya Y, Tamai K, Nevins JR. Multiple Rasdependent phosphorylation pathways regulate Myc protein stability. Genes Dev. 2000;14:2501-14.

48. Dai MS, Jin Y, Gallegos JR, Lu H. Balance of Yin and Yang: ubiquitylationmediated regulation of p53 and c-Myc. Neoplasia. 2006;8:630-44.

49. Lammers WJ, van Buuren HR, Hirschfield GM, Janssen HL, Invernizzi P, Mason AL, et al. Levels of alkaline phosphatase and bilirubin are surrogate end points of outcomes of patients with primary biliary cirrhosis: an international follow-up study. Gastroenterology. 2014;147:1338-49 (quiz e1315).

50. Poupon R. Liver alkaline phosphatase: a missing link between choleresis and biliary inflammation. Hepatology. 2015;61:2080-90.

51. Haarhaus M, Brandenburg V, Kalantar-Zadeh K, Stenvinkel P, Magnusson P. Alkaline phosphatase: a novel treatment target for cardiovascular disease in CKD. Nat Rev Nephrol. 2017;13:429-42.

52. Peters E, Heemskerk S, Masereeuw R, Pickkers P. Alkaline phosphatase: a possible treatment for sepsis-associated acute kidney injury in critically ill patients. Am J Kidney Dis. 2014;63:1038-48.

53. Sardiwal S, Magnusson P, Goldsmith DJ, Lamb EJ. Bone alkaline phosphatase in CKD-mineral bone disorder. Am J Kidney Dis. 2013;62:810-22.

54. Muller P, Henn W, Niedermayer I, Ketter R, Feiden W, Steudel WI, et al. Deletion of chromosome $1 p$ and loss of expression of alkaline phosphatase indicate progression of meningiomas. Clin Cancer Res. 1999:5:3569-77.

55. Lee SI, Kim GT, Kim HJ, Park SH, Kim EC. NOD2 Mediates Odontoblast Differentiation and RANKL Expression. J Dent Res. 2014;93:678-84.

56. Brondello JM, Pouyssegur J, McKenzie FR. Reduced MAP kinase phosphatase-1 degradation after p42/p44MAPK-dependent phosphorylation. Science. 1999;286:2514-7.

\section{Publisher's Note}

Springer Nature remains neutral with regard to jurisdictional claims in published maps and institutional affiliations.

Ready to submit your research? Choose BMC and benefit from:

- fast, convenient online submission

- thorough peer review by experienced researchers in your field

- rapid publication on acceptance

- support for research data, including large and complex data types

- gold Open Access which fosters wider collaboration and increased citations

- maximum visibility for your research: over $100 \mathrm{M}$ website views per year

At BMC, research is always in progress.

Learn more biomedcentral.com/submissions 